\title{
Freizügigkeit und Sozialpolitik - gibt es Fortschritt im Bereich des europäischen Arbeits- und Gesundheitsschutzes?
}

\author{
JAN CREMERS
}

Im Vertrag zur Gründung der Europäischen Wirtschaftsgemeinschaft (1957) unterstrich schon Artikel 117 den Willen der Mitgliedstaaten, , ,auf eine Verbesserung der Lebens- und Arbeitsbedingungen der Arbeitskräfte hinzuwirken und dadurch auf dem Weg des Fortschritts ihre Angleichung zu ermöglichen" (heute Art. 151 AEUV). Die Freizügigkeit, so hei $\beta t$ es, erfordere die Schaffung einer harmonisierten Gesetzgebung, um für alle Arbeitskräfte den gleichen (Mindest-)Schutz zu gewährleisten. Doch die EU-Kommission fördert zwar Freizügigkeit und grenzüberschreitende Arbeit, zeigt jedoch gegenwärtig in der Arbeitsschutzpolitik weitgehende Abstinenz. Während die EU-Rahmenstrategie zum Arbeits- und Gesundheitsschutz 2007 - 2012 noch Migranten neben jungen, alten und von Arbeitslosigkeit bedrohten Arbeitnehmern als eine der besonders gefährdeten Zielgruppen benannte und die Migration innerhalb und nach Europa als eine der drei zentralen Herausforderungen für den Arbeitsschutz verstand, ist in der im Juni 2014 verabschiedeten EU-Arbeitsschutzstrategie 2014-2020 jeder Bezug auf besonders gefährdete Arbeitnehmergruppen gestrichen und wird „Migration“ nicht mehr als Thema genannt, obwohl die Förderung der Mobilität zu den Hauptanliegen der Strategie Europa 2020 gehört. Als Schwerpunkte im Bereich des Arbeitsschutzes geblieben sind allein noch die Alterung der Erwerbsbevölkerung, die Prävention von Berufskrankheiten sowie die Umsetzung des Arbeitsschutzes in klein- und mittelständischen Betrieben, Letzteres unter der Maßgabe, dass bürokratische Belastungen zu minimieren seien. Während das Vorläuferprogramm noch unterstrich, dass eine Regulierung auf keinen Fall zu einer Absenkung des Schutzniveaus führen darf, heißt es in der neuen Arbeitsschutzstrategie 2014-2020, dass die Kommission zur Beseitigung unnötigen Verwaltungsaufwands für Klein- und Mittelbetriebe (KMU) die Vereinfachung der bestehenden Rechtsvorschriften anstrebt, während ein hohes Sicherheits- und Gesundheitsniveau jedoch gleichzeitig gewahrt werden soll.

Meine Schlussfolgerung ist, dass sich die europäische Arbeitsschutzpolitik als nicht mehr binnenmarktbeständig erweist. Das Generaldirektorat Beschäftigung, Soziales und Integration muss sich gegen andere Direktorate der Kommission zur Wehr setzen, die auf Wettbewerbsfähigkeit und Marktdenken fokussieren und mit dem Schlagwort „Bürokratieabbau" einen Vorwand gefunden haben, auch in die
Bestände der Sozialpolitik, hier namentlich der Arbeitsschutzpolitik, einzugreifen. Die jüngste Entwicklung soll nachfolgend kurz skizziert werden, bevor ich auf das besonders problematische Thema des unzureichenden Arbeitsschutzes für Migranten zu sprechen komme.

\section{Zum Hintergrund}

Zur Erinnerung: Die Einheitliche Europäische Akte (EEA) schuf 1985 die Grundlage für den Binnenmarkt, zugleich legte sie neue Zuständigkeiten der Gemeinschaft in der Sozialpolitik fest. Das angestrebte Gleichgewicht zwischen Wirtschaft und Sozialem wurde im Maastrichter Vertrag in Reaktion auf die Opposition Großbritanniens in einem gesonderten Sozialabkommen und Sozialprotokoll festgeschrieben. In Teilbereichen waren im EU-Rat nun qualifizierte Mehrheitsabstimmungen ausreichend, anstelle der bis dahin geforderten Einstimmigkeit bei Beschlüssen. Das Sozialabkommen wurde zu einer neuen Grundlage für eine europäische Arbeitsschutzpolitik, insbesondere im Hinblick auf die Festlegung arbeitsrechtlicher Mindestnormen. Im Politikfeld Arbeitsschutz entwickelte sich eine neue Dynamik - vielfach eher unbemerkt vorangetrieben von Verwaltungsfachleuten in Kooperation mit den Gewerkschaften. Eine Vielzahl von Einzelrichtlinien (mittlerweile rund 25) und die 1989 verabschiedete Rahmenrichtlinie zum Arbeits- und Gesundheitsschutz (89/391/EWG) schufen eine gemeinsame Rechtsbasis, die in nationales Recht umgesetzt werden musste. Sie lösten in den Mitgliedstaaten eine neue Dynamik aus, die zu wichtigen Verbesserungen der Regelwerke führte.

Demgegenüber zeigte die Kommission in den vergangenen Jahren im Bereich Arbeitsschutz weitgehende Abstinenz. Einige Richtlinienentwürfe, an denen noch bis 2013 gearbeitet wurde, wurden gestoppt oder verworfen, die Verabschiedung der neuen Arbeitsschutzstrategie 2014- 2020 verzögerte sich bis zum Sommer letzten Jahres. Zurückzuführen ist dies auf diverse Akteure, die unter dem Vorzeichen des Bürokratieabbaus und „besserer Regulierung “ einen starken Hang zu einer als Abbau sozialer Standards verstandenen Deregulierung zeigen.

Eine besondere Rolle kommt der 2007 eingesetzten High-Level Group on Administrative Burdens (HLG) (Sto- 
iber-Kommission) zu, die Möglichkeiten zum Bürokratieabbau in insgesamt 13 Politikfeldern auszuformulieren hatte. Die High Level Group stützte sich dabei u. a. auf zwei Studien zur Messung administrativer Kosten der EU-Gesetzgebung für Unternehmen, die von den Unternehmensberatungsagenturen Capgeminini/Deloitte/Ramboll Management ${ }^{1}$ verfasst wurden. Die Methodik (Standard Cost Model - SCM), die Repräsentativität der Datengrundlage und die Bewertung kann man mit Fragezeichen versehen. So konzentrierte sich die Analyse nur auf die verwaltungsbezogenen Tätigkeiten, die im Rahmen der Regeln eingehalten werden müssen, und nicht auf die Vorteile, die sich aus der Gesetzgebung ergeben. Das Sample bezog sich auf nur sechs Mitgliedstaaten kombiniert mit älteren Daten aus vier weiteren Ländern. Die Ergebnisse wurden hochgerechnet auf die Gesamtheit der Mitgliedstaaten. Datengrundlage bildeten face-to-face Gespräche mit Unternehmern. Die jeweilige Grundgesamtheit bleibt im Dunkeln. Eindeutig ist, dass es sich um Einstellungsmessungen handelte. Gemessen wurde die Höhe der Irritation der Unternehmer über die Maßnahme. Aus dieser Methodik harte Zahlen zu destillieren, ist problematisch, doch so manche Zahl wird mittlerweile als Wahrheit gehandelt und ständig wiederholt.

Die High Level Group legte im Oktober 2014 ihren Abschlussbericht vor. Er plädiert für eine „schlanke Umsetzung" von EU-Vorgaben und empfiehlt u.a. (Recommendation 11), dass nationale Gesetzgebung, die über den EU-seitig gesetzten Mindeststandard hinausgeht (gold-plating) explizit begründet werden muss. Dies verkehrt die Logik der Arbeitsschutzrahmenrichtlinie von 1989 ins Gegenteil; diese verstand Mindeststandards als Untergrenze und ließ günstigere nationale Regelungen explizit (Art. 13) unberührt, um eine Angleichung nach oben zu forcieren. Prioritäres Anliegen der Stoiber-Kommission ist jedoch die Wettbewerbsfähigkeit, nicht der Arbeitnehmerschutz.

Vor diesem Hintergrund erschließt sich, warum die neue EU-Arbeitsschutzstrategie nur drei Schwerpunkte setzt und besonders gefährdete Arbeitnehmergruppen nicht mehr erwähnt werden.

\section{Freizügigkeit und Arbeitnehmerschutz}

Mehrere Studien deuten darauf hin, dass besondere Präventionsmaßnahmen für Arbeitnehmer mit Migrationshintergrund nötig sind. Die European Agency for Occupational Safety and Health unterscheidet zwischen ausländischen Beschäftigten in hochqualifizierten Tätigkeiten (ICT, Kultur, Finanzwesen, etc) und Arbeitsmigranten sowie Wanderarbeitnehmern, die in risikoreichen Branchen (Bau, Landwirtschaft, Verkehr/Logistik, Gesundheitswesen, private Haushalte) tätig und an Arbeitsplätzen beschäftigt sind, an denen mangelnde Sprachkenntnisse und geringe Qualifikationen zu einer besonderen Gefährdung führen können. Neueinsteiger im Betrieb haben ein etwa 50 \% höheres Unfallrisiko. ${ }^{2}$ Es besteht ein negativer Zusammenhang zwischen zeitlich begrenzten und vorübergehenden Arbeitstä- tigkeiten und Arbeitsschutz. Wenn Migranten in Bereichen und Tätigkeiten arbeiten, in denen der Zeitdruck hoch ist und keine ausreichende Einarbeitung erfolgt, erhöht sich das Risiko weiter. ${ }^{4}$ OSHA-Bilbao weist auf drei besorgniserregende Probleme hin:

- eine hohe Beschäftigungsrate und vorrangiges Arbeiten in Hochrisikosektoren,

- sprachliche und kulturelle Barrieren bei der Kommunikation und bei Schulungsmaßnahmen im Bereich Sicherheit und Gesundheitsschutz.

- Zudem machen Wanderarbeitnehmer häufig Überstunden und/oder sind in schlechter gesundheitlicher Verfassung und daher auch anfälliger für arbeits- und berufsbedingte Verletzungen und Krankheiten. ${ }^{5}$

Dazu kommt, dass der Zugang zu lokalen Gesundheitseinrichtungen zwar rein rechtlich vorhanden, aber in der Praxis gegebenenfalls blockiert ist. Eine britische Studie zum Gesundheitswesen, einem Sektor der viele ausländische Arbeitnehmer und Arbeitnehmerinnen beschäftigt, zeigte auf, dass für Leiharbeiter und Arbeitsmigranten „krank werden“ keine Option darstellt, denn erstens geht damit das Risiko einher, den Job zu verlieren, zweitens verringert eine Krankschreibung die Chance wieder, nachgefragt zu werden und drittens kann sie - falls der Beschäftigte nicht ordentlich gemeldet ist - den Verlust jeden Einkommens bedeuten, da kein Krankengeld gezahlt wird. Die Risiken sind klar, man beißt auf die Zähne und macht weiter, ohne Rücksicht auf die Schutzmaßnahmen oder die eigene Gesundheit. ${ }^{6}$ Gleichzeitig wurde nachgewiesen, dass die notwendige Einarbeitung und Unterrichtung der vor Ort geltenden Regeln und Arbeitsweisen fehlten. Daraus folgen sowohl er-

1 Capgemini, Deloitte, Ramboll Management (2009): Final Report on Modules 3\&4 for Working Environment Priority Area; Capgemini, Deloitte, Ramboll Management (2010): EU Project on baseline measurement and reduction of administrative costs: Final report, incorporating report on Module 5.2 - Development of Reduction Recommendations.

2 http://www.arbeitsschutz-portal.de/beitrag/asp_news/3530/ strukturierte-einarbeitung-so-klappts-auch-mit-dem-neuen.html.

3 Quinlan, M./Mayhew, C./Bohle, P. (2001): The global expansion of precarious employment, work disorganisation and occupational health: A review of recent research, in: International Journal of Health Services 31 (2), S. 335-441.

4 Boege, K. (2012): Vorstudie zum Einfluss der Migration auf die Präventionstätigkeit in der Deutschen gesetzlichen Unfallversicherung, Institut für Arbeit und Gesundheit der DGUV, Abteilung Internationale Kooperation, Berlin

5 https://osha.europa.eu/de/priority_groups/migrant_workers.

6 Monteiro, B. (2014): Portuguese construction workers in Spain: Situated practices and transnational connections in the European field of construction (2003-2013), CLRNews 2-2014, Brüssel. 
höhte Risiken für die Mitarbeiter und Mitarbeiterinnen als auch für die Patienten. ${ }^{7}$

Die Bauindustrie ist und bleibt eine Branche mit „hoher Migrantenintensität" und mit Arbeitsverhältnissen, die gekennzeichnet sind durch zeitlich befristete Verträge und ortsveränderliche, vorübergehende Arbeitsplätze. Es gibt eine breite Palette von Arbeitsvertragsformen und besonders auch bei Arbeitern aus den beigetretenen Ländern ist die Quote der (Schein-)Selbstständigkeit hoch. ${ }^{8}$ Englische Forscher wiesen schon vor einigen Jahren eine hohe Frequenz tödlicher Unfälle am Arbeitsplatz unter Arbeitsmigranten nach. ${ }^{9}$

Die Europäische Entsenderichtlinie regelt die Gleichberechtigung der entsandten Arbeitnehmer im Hinblick auf den gesetzlichen Arbeits- und Gesundheitsschutz. Doch entsandte Beschäftigte sind besonderen Gefährdungen ausgesetzt. Bei der vom Autor angefertigten Entsendestudie wurden fragwürdige „legale“ Entsendepraktiken aufgezeigt, im Rahmen derer die rekrutierten Arbeitskräfte mit nicht vorhandener ordnungsgemäßer Sozialversicherung, nicht bezahlten Überstunden, Abzügen für Verwaltungskosten, erzwungenen und ungerechten Abzügen für Unterkunft und Transport, Steuerabzügen und Rückerstattungspflicht (nach der Rückkehr in die Heimat) von Lohnzahlungen konfrontiert wurden. In Extremfällen waren die Arbeitnehmer exzessiven Überstunden sowie der Nichteinhaltung von Ruhetagen (oder nur 1 bis 2 Tagen pro Monat) in Verbindung mit grundlegenden Arbeitsschutzproblemen ausgesetzt. Derartige Praktiken stellen eine klare Verletzung der Entsendevorschriften dar. Die Frage ist dann aber, wie Arbeitnehmer ihr Recht durchsetzen können. ${ }^{10}$

Unter den nicht eingehaltenen Arbeitsnormen bei entsandten Arbeitnehmern wurden insbesondere auch Fragen des Arbeitsschutzes aufgelistet: höhere Risiken bedingt durch Erschöpfung, kein Schulungsangebot, keine Übersetzung der Arbeitsschutzvorschriften, Mangel an erforderlicher Schutzausrüstung, ungesundes Arbeitsumfeld.

Die Einhaltung der Entsenderichtlinie zu kontrollieren, erweist sich als besonders schwierig. So zeigte etwa ein Bericht irischer Arbeitsschutzbehörden, dass, obwohl alle Arbeitgeber aufgefordert sind, der Arbeitsschutzbehörde (HSA) mitzuteilen, wenn Arbeiten auf einer neuen Bau- stelle aufgenommen werden, bei der zuständigen irischen Behörde DETI nur äußerst wenige Mitteilungen von Entsendeunternehmen eingehen. Ihrerseits stellten die irischen Behörden in diesem Zeitraum so gut wie keine Auskunftsanträge bei den zuständigen Institutionen in anderen Mitgliedstaaten. ${ }^{11}$

Eine Untersuchung des Autors zu diesem Thema zeigte, dass aufseiten der Erbringer grenzüberschreitender Dienstleistungen große Unwissenheit hinsichtlich der Sozialschutzvorschriften, einschließlich der Gesundheits- und Sicherheitsfragen besteht. ${ }^{12}$ Qualitative Forschung, die auf Interviews mit entsandten Beschäftigten beruht, hat gezeigt, dass diese ihrerseits über ihre Rechte weitgehend uninformiert sind. ${ }^{13}$ Ein Projekt des Französischen Dachverbandes der Arbeitsinspektoren INTEFP stellte z. B. fest, dass es für Wanderarbeiter und sonstige Arbeitsmigranten an wichtigen Arbeitsschutzinformationen fehlt und vorhandene online-Informationen unzureichend sind, wenn es um notwendige Information vor Ort geht. Die Inspektoren berichten weiter über Nicht-Einhaltung der Vorschriften, Diskriminierung von Migranten und mangelnde Koordination zwischen Unternehmen, die mit entsandten Arbeitnehmern gleichzeitig an einem Arbeitsplatz tätig sind.

Das Projekt CIBELES, durchgeführt vom Senior Labour Inspectors Committee (SLIC) von 2010 bis 2011, bestätigte, dass Verstöße gegen Entsenderegeln nicht vollständig untersucht werden konnten, und dass es wichtige Behinderungen gab bei der grenzüberschreitenden Vollstreckung von Geldstrafen und anderen Sanktionen. Arbeitsinspektoren haben große Schwierigkeiten, entsandte Arbeitnehmer oder Selbstständige am Arbeitsplatz zu identifizieren und die notwendigen Unterlagen sind oft nicht vorhanden. Infolge dessen bleibt vieles verdeckt und/oder ungestraft.

$\mathrm{Zu}$ berücksichtigen ist auch, dass anders als bei aus EUStaaten entsandten Arbeitnehmern, die per Gesetz durch die Gewährleistung von (Mindest-)Arbeits- und Beschäftigungsbedingungen geschützt sind (welche zumindest Aspekte wie Arbeitszeiten, Mindestlöhne, Beurlaubung, Arbeitsschutz umfassen), die geltenden arbeitsrechtlichen Bestimmungen keine Anwendung finden auf aus Drittstaaten entsandte Arbeitnehmer.
7 Maroukis, T. (2015): Stretching the flexible labour:Temporary agency work and 'bank' labour in the lower skill echelons of the healthcare labour market in UK and Greece, in: Journal of European Social Policy (im Erscheinen).

8 Harvey, M./Behling, F. (2008):The evasion economy: False self-employment in the UK construction industry, UCATT, London, http://ucatt.infobo.co.uk/sites/default/files/uploaded/publications/Evasion-Economy-UCATT.pdf.

9 Centre for Corporate Accountability (CCA) (2009): Migrants' workplace deaths in Britain, London, http://www. corporateaccountability.org.uk/dl/HSE/migrant/cca_irwinmitchell.pdf.
10 Cremers, J. (2013): Free provision of services and crossborder labour recruitment, in: Policy Studies 34 (2), S. $201-220$

11 Cremers, J. (2011): In search of cheap labour in Europe. Working and living conditions of posted workers, CLR/ibooks, Utrecht/Brüssel.

12 Cremers, J./Donders, P. (2004): The free movement of workers in the European Union, CLR/Reeds Business Information, Brüssel.

13 Wagner, I. (2014): Rule enactment in a pan-European labour market:Transnational posted work in the German 
Gerade wegen der Bedeutung der Untervergabe und der Beschäftigung von entsandten Arbeitnehmern und Leiharbeitnehmern ist die von der Baustellenrichtlinie (92/57/EWG) vorgeschriebene Koordinierung zwischen allen Beteiligten auf Baustellen von größter Bedeutung. Auch Selbstständige und Arbeitgeber, die selbst eine berufliche Tätigkeit auf einer Baustelle ausüben, sollten (gemäß Artikel 6.b der Richtlinie) eingebunden werden und den vorgesehenen Sicherheits- und Gesundheitsschutzplan anwenden. Nicht umsonst sagt die Richtlinie aus, dass ab der Vorbereitung des Bauprojekts, jedoch auch während der Durchführung der Bauarbeiten, eine verstärkte Koordinierung zwischen den verschiedenen Ausführenden erforderlich ist. Die dazu gehörende obligatorische gegenseitige Informationspflicht, unter Einbeziehung der Selbstständigen, ist eine notwendige Voraussetzung für das Funktionieren der Koordinierung, eines der grundlegenden Prinzipien der Richtlinie. ${ }^{14}$ Man kann deshalb zu der Schlussfolgerung kommen, dass das Rechtsinstitut der Kettenhaftung, das alle Unternehmen auf einer Baustelle miteinander verbindet, und die Aufstellung einer Datei mit entsprechenden Informationen über Arbeitsschutz und Sicherheitsfragen für alle stattfindenden und nachfolgenden Arbeiten eine Normalität in Europa, also business-as-usual, sein sollte.

Nicht so für die oben erwähnten Berater Capgemini/ Deloitte/Ramboll der Stoiber-Kommission. Diese stellen auf der Grundlage ihrer Interviews fest, dass die Koordinierungsverpflichtung aus Sicht der Unternehmer zu großen Irritationen führe und als eine der größten Verwaltungslasten zur Disposition zu stellen sei.

\section{Fazit - Grundrecht oder Produktionsfaktor?}

Die EU fördert die Mobilität und erwartet in den nächsten Jahrzehnten eine große Netto-Immigration aus Drittländern. Wanderarbeit kann angesichts einer alternden Erwerbsbevölkerung einer der bestimmenden Faktoren für das Funktionieren des EU-Arbeitsmarktes werden. Migranten werden aber bis jetzt hauptsächlich in arbeitsintensiven, gefährlichen und schlecht bezahlten, sogenannten 3D-Berufen („dirty, dangerous, difficult“) beschäftigt. ${ }^{15}$

Entsandte Arbeitnehmer sind oft Arbeitnehmer, die am „Boden“ des Arbeitsmarktes beschäftigt sind, wo Arbeitsschutznormen umgangen werden. Die Gestaltung des Binnenmarktes, gestützt auf wirtschaftliche Freiheiten (wie die Freizügigkeit und die freie Dienstleistung), gefährdet so die Gesundheit derer, die eigentlich als Träger und Verkörperung dieser Binnenmarktwerte zu betrachten sind.

Deswegen sind in den nächsten Jahren weiterführende Arbeitsschutzkonzepte am Arbeitsplatz zu entwickeln. Die Gesundheit der Arbeitnehmer darf niemals vernachlässigt werden wegen politischer Apathie, oder, noch schlimmer, zu einem Spielball zwischen Ländern im Wettbewerb und von kommerziellen Interessen werden. So ist auch für die Internationale Arbeitsorganisation (IAO/ILO) Arbeitsschutz ein integraler Bestandteil der gesamten Arbeit, mit einem weiteren Fokus auf unsichtbare und gefährdete Gruppen von Arbeitnehmern in der informellen Wirtschaft, Agrarwirtschaft und Wanderarbeiter. Eine Sensibilisierung in diesem Bereich ist dringend erforderlich. ${ }^{16}$

\section{AUTOR}

JAN CREMERS, Dr. h.C., ist Wissenschaftler am Amsterdamer Institut für Arbeitsstudien (AIAS) und am European Institute for Construction Labour Research (CLR). Arbeitsschwerpunkte: Arbeitsbedingungen/Arbeitsschutz (Bausektor, Migration) und Corporate Governance.

j.cremers@uva.nl construction sector, online Veröffentlichung des British Journal of Industrial Relations, http://onlinelibrary.wiley. com/doi/10.1111/bjir.12053/abstract.

14 Gemäß Artikel 5.c der Richtlinie 92/57/EWG fasst der Koordinator eine Unterlage zusammen, die den Merkmalen des Bauwerks Rechnung trägt und die zweckdienliche Angaben in Bezug auf Sicherheit und Gesundheitsschutz, die bei eventuellen späteren Arbeiten zu berücksichtigen sind, enthält. Selbstständige haben die Hinweise der Sicherheits und Gesundheitsschutzkoordinatoren zu berücksichtigen.

15 Internationale Arbeitsorganisation (IAO) (2011): Research on occupational safety and health for migrant workers, Genf.

16 Aussage von IAO General Direktor Guy Rider, XX. Weltkongress für Sicherheit und Gesundheit bei der Arbeit 2014, Frankfurt a. M.
(1) 